\title{
Desempenho dos Centros de Especialidades Odontológicas frente ao quadro sociodemográfico dos municípios do Amazonas, Brasil, 2009
}

Performance of Specialized Dental Care Centers considering the sociodemographic context of municipalities of the Amazonas State (Brazil, 2009)

Fernando José Herkrath', Ana Paula Corrêa de Queiroz Herkrath², Lívea Nancy Bulcão da Silva Costa3', Maria Jacirema Ferreira Gonçalves ${ }^{4}$

Mestre em Saúde, Sociedade e Endemias na Amazônia pela Universidade Federal do Amazonas (UFAM) - Manaus (AM). fernandoherkrath@gmail.com

Doutoranda em Saúde Pública pela Escola Nacional de Saúde Pública pela Fundação Oswaldo Cruz (FIOCRUZ) - Rio de Janeiro (RJ). anapaulaqueiroz@gmail.com

${ }^{3}$ Mestre em Saúde, Sociedade e Endemias na Amazônia pela Universidade Federal do Amazonas (UFAM) - Manaus (AM).

livea_nancy@hotmail.com

${ }^{4}$ Doutora em Saúde Coletiva pela Universidade do Estado do Rio de Janeiro (UERJ) - Rio de Janeiro (RJ). Professora da Escola de Enfermagem da Universidade Federal do Amazonas (UFAM) - Manaus (AM). Professora do Programa de Pós-Graduação Multi-institucional em Saúde, Sociedade e Endemias na Amazônia da Universidade Federal do Amazonas (UFAM)/Centro de Pesquisas Leônidas e Maria Deane (CPqLMD - FIOCRUZ)/Universidade Federal do Pará (UFPA). Professora do Programa de PósGraduação Mestrado em Associação Ampla em Enfermagem da Universidade Federal do Pará (UFPA)/ da Universidade Federal do Amazonas (UFAM).

jaciremagoncalves@ufam.edu.br
RESUMO O estudo avaliou o cumprimento das metas de desempenho dos Centros de Especialidades Odontológicas (CEO) do estado do Amazonas, utilizando dados secundários do SIA-SUS, considerando as variáveis estruturais dos estabelecimentos, cobertura da atenção básica e indicadores sociodemográficos dos municípios. Treze CEO foram avaliados. Um estabelecimento de Manaus e os de Maués e Parintins apresentaram melhor desempenho em relação ao Cumprimento Global das Metas. A cobertura das Equipes de Saúde Bucal na atenção básica parece não acompanhar o desempenho dos estabelecimentos. Os resultados sugerem que o desempenho dos CEO está relacionado às características sociodemográficas dos municípios.

PALAVRAS CHAVE: Serviços de Saúde Bucal; especialidades odontológicas; sistemas de informação.

\begin{abstract}
The study evaluated the compliance of performance goals of the Centers for Dental Specialties of the state of Amazonas, using secondary data from the SIA-SUS, considering the structural variables of establishments, comprehensiveness of basic health care and sociodemographic indicators of municipalities. Thirteen centers were assessed. One establishment of Manaus and the Maués and Parintins showed better performance in relation to the global compliance of goals. Coverage of Oral Health Teams in primary care seems not to follow the performance of establishments. The results suggest that the performance of the Centers is related to the sociodemographic characteristics of the municipalities.
\end{abstract}

KEYWORDS: Dental Health Services; dental specialties; information systems. 


\section{Introdução}

A realidade da saúde bucal da população brasileira, apesar do progresso recente, caracteriza-se por ser ainda insatisfatória e abaixo dos níveis aceitáveis e preconizados pela Organização Mundial de Saúde. Mesmo que o país tenha dado um passo importante para a reduçáo da prevalência da doença cárie na faixa etária de 12 anos, por meio de açóes e atividades preventivas, grande parte da população adulta e idosa sofre ainda das mazelas dessa doença e de outras doenças orais. Aproximadamente $56 \%$ dos idosos são completamente edêntulos, o que sugere disponibilidade limitada dos serviços odontológicos, em especial dos serviços de atenção secundária (PINHEIRO; TORRES, 2006; BRASIL, 2004a). Até o lançamento do programa Brasil Sorridente, em 2004, a assistência odontológica restringia-se quase que exclusivamente aos serviços básicos, com menos de 3,5\% dos atendimentos odontológicos feitos no Sistema Único de Saúde (SUS) correspondentes a tratamentos especializados, demonstrando a marginalização e escassez dos serviços de atenção secundária (BRASIL, 2004b).

Para fazer frente ao desafio de ampliar e qualificar a oferta de serviços odontológicos especializados, o Ministério da Saúde definiu, inicialmente por meio das Diretrizes da Política Nacional de Saúde Bucal, a implantação e melhoria de Centros de Referência de Especialidades Odontológicas, os atuais Centros de Especialidades Odontológicas (CEO), regidos pelas normas estabelecidas na Portaria no 1.570/GM, de 29 de julho de 2004 (BRASIL, 2004c), que aborda também os requisitos necessários para a implantação e credenciamento dos CEO. Esses centros caracterizam-se por serem unidades de referência para as equipes de Saúde Bucal da atenção básica, estando sempre integrados ao processo de planejamento locorregional, ofertando, de acordo com a realidade epidemiológica de cada região e município, procedimentos clínicos odontológicos complementares aos realizados no nível da atenção básica, com o objetivo de combater a perda de tempo, a longa espera por procedimentos e a falta de resoluçáo dos problemas enfrentados pelos usuários do SUS, que necessitavam de tratamento odontológico integral. Entre os procedimentos especializados ofertados pelos CEO incluem-se tratamentos cirúrgicos periodontais, tratamento endodônticos e de dentística de maior complexidade, além de procedimentos cirúrgicos compatíveis com esse nível de atenção (ORGANIZAÇÃO PANAMERICANA DE SAÚDE; BRASIL, 2006).

Todavia, em algumas regióes do Brasil, a implantação desses centros ocorre de maneira ainda lenta frente à demanda da população por tais serviços. $\mathrm{O}$ estado do Amazonas, que possui 62 municípios, oferta poucos serviços públicos especializados em saúde bucal, apesar das necessidades explicitadas no levantamento epidemiológico de âmbito nacional em saúde bucal para a região (BRASIL, 2004a). Dados do Cadastro Nacional de Estabelecimentos de Saúde (CNES) do Ministério da Saúde mostram que apenas 1,3\% dos 832 CEO credenciados no Brasil até o início de 2010 localizavam-se no Amazonas, representando um estabelecimento para aproximadamente 250.000 habitantes, enquanto estados de porte populacional semelhante - embora de menor extensão territorial - como Alagoas, Rio Grande do Norte, Piauí e Paraíba abrigavam, respectivamente, $2,0 \%, 2,5 \%, 3,1 \%$ e $5,2 \%$ destes estabelecimentos. Nesse cenário, torna-se primordial o conhecimento quantitativo e qualitativo dos dados referentes à atenção secundária, para que possa servir de base para conhecimento e melhor organização do serviço, ao mesmo tempo em que se torna possível o acompanhamento e monitoramento dos serviços. Um importante instrumento que pode ser utilizado com este objetivo é o Sistema de Informação Ambulatorial do Sistema Único de Saúde (SIA-SUS), que registra o quantitativo de procedimentos realizados pelos estabelecimentos de saúde.

Este estudo teve, portanto, o objetivo de caracterizar a atenção especializada em saúde bucal no estado do Amazonas, considerando as características sociodemográficas dos municípios que ofertavam o serviço, no ano de 2009.

\section{Método}

Estudo quantitativo e descritivo no qual foram utilizados dados secundários oriundos do banco de dados do Sistema de Informaçōes Ambulatoriais do Sistema 
Único de Saúde (SIA-SUS), disponíveis no Departamento de Informática do Sistema Único de Saúde (Datasus), com o intuito de obter a quantidade aprovada de procedimentos odontológicos ambulatoriais provenientes dos 13 CEO do estado do Amazonas cadastrados no CNES, referentes ao período de janeiro a dezembro de 2009.

Os arquivos com a produção ambulatorial mensal do estado do Amazonas foram obtidos no Datasus ${ }^{1}$, os quais foram tabulados por meio do software TabWin, versão $3.5^{2}$, sendo filtrados os procedimentos da produção ambulatorial mensal de cada CEO cadastrado.

Após a tabulação, os dados foram exportados para o programa Microsoft Office Excel $^{\circledR} 2003$, onde foi feita organizaçáo dos procedimentos de acordo com os subgrupos: procedimentos básicos, procedimentos de periodontia, procedimentos de endodontia e procedimentos de cirurgia oral menor. Tais subgrupos foram assim organizados tendo como base a Portaria no 600/ GM, de 23 de março de 2006, que estabelece as metas de desempenho a serem alcançadas para cada tipo de CEO (BRASIL, 2006b).

Também em 23 de março de 2006, a Portaria no 599/GM (BRASIL, 2006a), em substituição à no 1.570/GM (BRASIL, 2004c), definiu as características das modalidades de CEO. Dessa forma, foram classificados em Tipo I os que possuíam três cadeiras odontológicas; Tipo II os que tinham de quatro a seis cadeiras odontológicas; e Tipo III os que possuíam mais de sete cadeiras odontológicas. Todos os dados estruturais dos CEO analisados foram obtidos no CNES.

Para avaliação de desempenho foi utilizado o indicador desenvolvido por Figueiredo e Goes (2009) denominado Cumprimento Global das Metas (CGM), correspondente ao quociente entre a quantidade total de procedimentos realizados durante $\mathrm{n}$ meses de cada subgrupo de especialidades odontológicas pelo número de procedimentos correspondente à meta deste subgrupo por $\mathrm{n}$ meses, multiplicado por 100. Quando o cumprimento percentual da meta normatizada para cada subgrupo de procedimentos odontológicos especializados foi igual ou superior a $100 \%$, considerou-se meta atingida. Conforme estabelecido pelo indicador CGM, o desempenho dos serviços foi classificado em: desempenho ruim (CEO que não cumpriram nenhuma ou apenas uma das metas); desempenho regular (CEO que cumpriram duas metas); desempenho bom (CEO que cumpriram três metas); e desempenho ótimo (CEO que cumpriram todas as quatro metas).

A meta mensal por subgrupo para cada tipo de Centro de Especialidades Odontológicas foi definida pela Portaria no 600/GM (BRASIL, 2006b):

a) Centro de Especialidades Odontológicas tipo I - 80 procedimentos do subgrupo básico; 60 procedimentos do subgrupo periodontia; 35 procedimentos do subgrupo endodontia; e 80 procedimentos do subgrupo cirurgia oral menor;

b) Centro de Especialidades Odontológicas tipo II - 110 procedimentos do subgrupo básico; 90 procedimentos do subgrupo periodontia; 60 procedimentos do subgrupo endodontia; e 90 procedimentos do subgrupo cirurgia oral menor; e,

c) Centro de Especialidades Odontológicas tipo III - 190 procedimentos do subgrupo básico; 150 procedimentos do subgrupo periodontia; 95 procedimentos do subgrupo endodontia; e 170 procedimentos do subgrupo cirurgia oral menor.

Na sequência, analisou-se o cumprimento das metas de acordo com as variáveis:

Estruturais dos CEO - tipo de CEO, presença de Laboratório Regional de Prótese Dentária (LRPD) e quantitativo de auxiliares de saúde bucal e cirurgióes-dentistas por CEO. Dados disponíveis no CNES. Quando houve divergência em relação à habilitação do estabelecimento no Ministério da Saúde, o tipo do CEO foi determinado pela infraestrutura cadastrada no CNES, módulo conjunto, equipamentos.

Reorganização da atençáo básica - cobertura populacional das equipes da Estratégia de Saúde da Família (ESF) e cobertura das Equipes de Saúde Bucal

Disponível em: <http://w3.datasus.gov.br/siasih/siasih.php>. Acesso em 16 jun 2010

2 BRASIL. Ministério da Saúde. Disponível em: <http://www2.datasus.gov.br/DATASUS/index.php?area=040805\&item=3>. Acesso em 16 jun 2010. 
(ESB) da ESF. O percentual de cobertura das equipes foi calculada com base nas metas definidas pela Portaria no 1329/GM, de 12 de novembro de 1999, e Portaria no 673, de 03 de junho de 2003: 1 ESF e 1 ESB para cada 3.450 habitantes (BRASIL, 1999; BRASIL, 2003). Aqueles municípios cujo cálculo da cobertura da ESF e ESB extrapolou o percentual total da população, por possuírem um número de equipes superior ao mínimo estipulado nas portarias, foram representados por um valor superior a $100 \%$, ilustrando a magnitude de tal situação e respeitandose, assim, o mesmo método de cálculo para todos os municípios.

Indicadores sociodemográficos dos municípios - população, Índice de Desenvolvimento $\mathrm{Hu}$ mano (IDH) municipal do ano de 2000, PIB per capita, analfabetismo, incidência de pobreza. O perfil dos municípios envolvidos foi obtido no banco de dados do Instituto Brasileiro de Geografia e Estatística (IBGE) ${ }^{3}$, com exceção do IDH municipal, consultado no site do Programa das Naçóes Unidas para o Desenvolvimento (PNUD) ${ }^{4}$.

A análise dos dados foi descritiva por meio da distribuição de frequência absoluta e percentual das variáveis, bem como das médias dos indicadores calculados. A distribuição dos indicadores conforme cada tipo de CEO e por município foi apresentada em tabelas e os estabelecimentos foram agrupados de acordo com o desempenho obtido e os valores médios, máximo e mínimo calculados para os indicadores socioeconômicos do município, cobertura das ESF e ESB e recursos humanos do CEO. O campo referente a cada variável avaliada não foi preenchido quando os estabelecimentos não possuíam os dados disponíveis.

Como foram utilizados dados secundários disponíveis ao acesso público, onde não constam dados de identificação, este estudo não necessitou de submissão ao Comitê de Ética em Pesquisa envolvendo seres humanos.

\section{Resultados}

Até dezembro de 2009, treze CEO haviam sido implantados no Amazonas, quatro na capital, Manaus, e nove no interior do estado, nos municípios de Boca do Acre, Iranduba, Itacoatiara, Lábrea, Maués, Parintins, Tefé e São Gabriel da Cachoeira, este último com dois CEO cadastrados. Destes nove municípios, quatro configuram-se como de referência regional (Itacoatiara, Lábrea, Parintins e Tefé), três como de referência microrregional (Boca do Acre, Maués e São Gabriel da Cachoeira) e Manaus como centro de referência macrorregional. $\mathrm{O}$ perfil demográfico, social e econômico dos municípios envolvidos no estudo está compendiado na Tabela 1.

A Tabela 2 reúne as características dos $\mathrm{CEO}$ do Amazonas avaliados. Segundo a infraestrutura cadastrada no CNES, cinco foram classificados como tipo I, sete como tipo II e apenas um como tipo III. Dentre o total de estabelecimentos, três não apresentavam habilitação no Ministério da Saúde, dois na capital Manaus e outro no município de São Gabriel da Cachoeira. Cinco dos treze CEO possuíam LRPD habilitado.

A avaliação do Cumprimento Global das Metas mostrou que o estabelecimento Manaus-2 do município de Manaus e os CEO de Maués e Parintins cumpriram todas as metas, recebendo a classificação ótimo, o estabelecimento Manaus-1 cumpriu três das quatro metas, recebendo a classificação bom, o estabelecimento Manaus-4, os dois de São Gabriel da Cachoeira e o do município de Tefé cumpriram apenas uma das metas, recebendo a classificação regular, e os estabelecimentos de Iranduba e Lábrea não cumpriram nenhuma das metas de produção definidas para cada subgrupo pelo Ministério da Saúde, recebendo a classificação ruim, conforme ilustra a Tabela 3. Os CEO dos municípios de Boca do Acre, Itacoatiara e um de Manaus não possuíam produção no SIA-SUS e não puderam ser avaliados.

Os estabelecimentos com pior desempenho quanto ao cumprimento das metas apresentaram

${ }^{3}$ Disponível em: < htpp://www.ibge.gov.br/cidadesat/default.php>. Acesso em: 19 ago 2010.

${ }^{4}$ Disponível em: <http://www.pnud.org.br/atlas/tabelas/index.php>. Acesso em 21 ago 2010. 
Tabela 1. Perfil dos municípios incluídos no estudo, Amazonas, Brasil, 2009

\begin{tabular}{lccllllll}
\hline MUNICÍPIO & POPULAÇÃO & IDH* & $\begin{array}{l}\text { *PIB PER } \\
\text { CAPITA }\end{array}$ & ANALABETISMO & $\begin{array}{l}\text { INCIDÊNCIA DE } \\
\text { POBREZA (\%) }\end{array}$ & $\begin{array}{l}\text { COBERTURA } \\
\text { ESF*** } \%)\end{array}$ & $\begin{array}{l}\text { COBERTURA } \\
\text { ESB*** (\%) }\end{array}$ \\
\hline $\begin{array}{l}\text { Boca do } \\
\text { Acre }\end{array}$ & 31221 & 0,611 & 5569 & 37 & 40,36 & 66,30 & 66,30 \\
\hline Iranduba & 33884 & 0,694 & 4639 & 20,8 & 63,52 & 183,27 & 152,73 \\
\hline Itacoatiara & 89440 & 0,711 & 7211 & 12,4 & 56,78 & 96,43 & 81,00 \\
\hline Lábrea & 39393 & 0,598 & 4776 & 41,3 & 61,04 & 70,06 & 52,55 \\
\hline Manaus & 1738641 & 0,774 & 20894 & 6,1 & 40,98 & 32,74 & 10,12 \\
\hline Maués & 49666 & 0,689 & 4090 & 15,4 & 55,25 & 76,41 & 65,52 \\
\hline Parintins & 107250 & 0,696 & 3518 & 9,4 & 60,07 & 77,20 & 35,38 \\
\hline $\begin{array}{l}\text { São } \\
\text { Gabriel da } \\
\text { Cachoeira }\end{array}$ & 41885 & 0,673 & 3621 & 23,2 & 44,4 & 32,95 & 32,95 \\
\hline \begin{tabular}{l} 
Tefé \\
\cline { 2 - 7 }
\end{tabular} & 64671 & 0,663 & 5078 & 20,6 & 61,64 & 69,35 & 37,34 \\
\hline
\end{tabular}

*Índice de Desenvolvimento Humano (IDH); Produto Interno Bruto (PIB); Estratégia de Saúde da Família (ESF); Equipes de Saúde Bucal (ESB).

** calculado pelo no de equipes × 3450 × 100 / população.

Fontes: IBGE; CNES; PNUD, 2000.

menor IDH e PIB per capita, maior índice de analfabetismo e incidência de pobreza, além de maiores percentuais de cobertura da ESF e ESB. A distribuição dos estabelecimentos segundo o desempenho obtido e os indicadores sociodemográficos do município, cobertura da ESF e ESB e recursos humanos do estabelecimento está descrita na Tabela 4 .

\section{Discussão}

O procedimento de criação e concretização do Sistema Único de Saúde (SUS) tem repercutido em amplos debates acerca da disposição e do planejamento dos serviços de saúde prestados à população brasileira, almejando tornar presentes no cotidiano dos usuários os princípios que regem o SUS: a universalização do acesso, a integralidade do tratamento e a equidade dos serviços (BALDANI et al., 2005). Nesse contexto de reestruturação do Sistema de Saúde, mais especificamente da atenção odontológica, destaca-se a criação dos CEO com o objetivo de ampliar o acesso a todas as esferas de atenção à saúde bucal e de reorganizar as açōes odontológicas básicas e especializadas. No estado do Amazonas, o número de CEO implantados até o final de 2009, quase cinco anos após a regulamentação da atenção secundária em saúde bucal (BRASIL, 2004c), pode ser considerado baixo em comparação à expansão destes serviços em outras Unidades da Federação (FIGUEIREDO; GOES, 2009; BRASIL; ORGANIZAÇÃO PAN-AMERICANA DA SAÚDE, 2010). Nesse período, apenas treze CEO estavam disponíveis para uma população de aproximadamente 2.800.000 habitantes. Os dados epidemiológicos disponíveis demonstram que o estado contava ainda com número insuficiente destes estabelecimentos, a despeito da alta demanda pelos serviços especializados em saúde bucal por parte da população (BRASIL, 2004a). 
Tabela 2. Características dos treze Centros de Especialidades Odontológicas avaliados, Amazonas, Brasil, 2009

\begin{tabular}{lcclllll}
\hline CEO & TIPO & $\begin{array}{c}\text { HABILITADO } \\
\text { COMO }\end{array}$ & $\begin{array}{l}\text { DATA DE } \\
\text { HABILITAÇÃO }\end{array}$ & $\begin{array}{l}\text { COMPETÊNCIA } \\
\text { INICIAL }\end{array}$ & *LRPD & $\begin{array}{l}\text { NÚMERO } \\
{ }^{*} \text { CD }\end{array}$ & $\begin{array}{l}\text { PROPORÇÃO } \\
{ }^{*} \text { CD/ASB }\end{array}$ \\
\hline Boca do Acre & II & II & $21 / 12 / 2009$ & $11 / 2009$ & NÃO & 4 & 0,8 \\
\hline Iranduba & II & II & $21 / 12 / 2009$ & $11 / 2009$ & NÃO & 8 & 1,33 \\
\hline Itacoatiara & I & II & $21 / 12 / 2009$ & $11 / 2009$ & NÃO & 8 & 2,66 \\
\hline Lábrea & I & I & $07 / 10 / 2009$ & $10 / 2009$ & NÃO & 5 & - \\
\hline Manaus-1 & II & II & $28 / 08 / 2007$ & $08 / 2007$ & NÃO & 10 & 1,11 \\
\hline Manaus-2 & II & - & - & - & NÃO & 16 & 1,77 \\
\hline Manaus-3 & II & - & - & - & NÃO & - & - \\
\hline Manaus-4 & III & III & $12 / 11 / 2008$ & $11 / 2008$ & SIM & 15 & 1,07 \\
\hline Maués & I & I & $30 / 03 / 2006$ & $03 / 2006$ & SIM & 4 & 1 \\
\hline Parintins & II & II & $19 / 01 / 2006$ & $01 / 2005$ & SIM & 7 & 1,75 \\
\hline $\begin{array}{l}\text { São Gabriel da } \\
\text { Cachoeira-1 }\end{array}$ & I & II & $19 / 012006$ & $01 / 2006$ & SIM & 3 & 1 \\
\hline $\begin{array}{l}\text { São Gabriel da } \\
\text { Cachoeira-2 }\end{array}$ & II & - & - & - & NÃO & 7 & 1,75 \\
\hline \begin{tabular}{l} 
Tefé \\
\hline
\end{tabular} & I & II & $25 / 08 / 2006$ & $05 / 2006$ & SIM & 6 & 3 \\
\hline
\end{tabular}

*Centro de Especialidades Odontológicas (CEO); Laboratório Regional de Prótese Dentária (LRPD); Cirurgião-dentista (CD); Auxiliar de saúde bucal (ASB).

Fonte: CNES; SIA-SUS

Quanto à habilitação dos estabelecimentos pelo Ministério da Saúde, destaca-se que três CEO ainda não a possuíam e outros três inexplicavelmente mostravam incompatibilidade entre o tipo de CEO habilitado e a estrutura física cadastrada no CNES. Assim, o estudo considerou na avaliação de desempenho os estabelecimentos de Iranduba, São Gabriel da Cachoeira-1 e Tefé como CEO tipo I, atendendo ao número de cadeiras odontológicas cadastradas no CNES, embora, mesmo assim, o desempenho destes não tenha sido satisfatório.

Segundo o indicador de CGM estabelecido, apenas três $\mathrm{CEO}$ alcançaram as quatro metas propostas, um no município de Manaus e os estabelecimentos localizados em Maués e Parintins. Outro CEO de Manaus obteve desempenho bom, referente ao cumprimento de três metas e os seis demais apresentaram desempenho regular ou ruim, considerando que três estabelecimentos não puderam ser avaliados por não apresentarem produção odontológica no SIA-SUS para o período avaliado, dois destes provavelmente por terem sido habilitados apenas no final do ano de 2009. Uma justificativa para o registro apenas de procedimentos ambulatoriais básicos e o consequente desempenho ruim apresentado pelos CEO de Lábrea e Iranduba poderia estar relacionada ao seu curto tempo de habilitação.

Evidenciou-se que 60\% dos CEO com dados disponíveis para avaliação não conseguiram realizar a totalidade mínima exigida para os procedimentos cirúrgicos e ainda $20 \%$ superaram a meta com uma média muito próxima ao limite estipulado. Uma possível explicação seria a possibilidade de estes procedimentos estarem sendo executados na atenção básica, sugerindo 
Tabela 3. Número de procedimentos especializados realizados mensalmente pelos CEO do estado do Amazonas durante o ano de 2009 confrontado com as metas estipuladas pelo Ministério da Saúde para cada tipo de CEO, Amazonas, Brasil, 2009

\begin{tabular}{|c|c|c|c|c|c|}
\hline \multirow{2}{*}{$\begin{array}{l}\text { MUNICÍPIO } \\
\text { Boca do Acre }\end{array}$} & \multicolumn{2}{|c|}{$\begin{array}{c}\text { MÉDIA MENSAL DE } \\
\text { PROCEDIMENTOS EM } 2009\end{array}$} & \multirow{2}{*}{$\begin{array}{c}\text { CUMPRIMENTO DAS } \\
\text { METAS (\%) } \\
-\end{array}$} & \multirow{2}{*}{$\begin{array}{c}\text { CUMPRIMENTO } \\
\text { GLOBAL } \\
-\end{array}$} & \multirow{2}{*}{$\begin{array}{c}\text { CLASSIFICAÇÃO } \\
-\end{array}$} \\
\hline & - & - & & & \\
\hline Iranduba & $\begin{array}{l}\text { Proc. Básicos } \\
\text { Periodontia } \\
\text { Endodontia } \\
\text { Cirurgia }\end{array}$ & $\begin{array}{c}278,6 \\
0 \\
0 \\
0 \\
\end{array}$ & $\begin{array}{c}253,3 \\
0 \\
0 \\
0 \\
\end{array}$ & $\begin{array}{l}\text { SIM } \\
\text { NÃO } \\
\text { NÂOO } \\
\text { NÂOO }\end{array}$ & RUIM \\
\hline Itacoatiara & - & - & - & - & - \\
\hline Lábrea & $\begin{array}{l}\text { Proc. Básicos } \\
\text { Periodontia } \\
\text { Endodontia } \\
\text { Cirurgia }\end{array}$ & $\begin{array}{c}654,3 \\
0 \\
0 \\
0\end{array}$ & $\begin{array}{c}817,9 \\
0 \\
0 \\
0\end{array}$ & $\begin{array}{l}\text { SIM } \\
\text { NÃO } \\
\text { NÂO } \\
\text { NÃO }\end{array}$ & RUIM \\
\hline Manaus-1 & $\begin{array}{l}\text { Proc. Básicos } \\
\text { Periodontia } \\
\text { Endodontia } \\
\text { Cirurgia }\end{array}$ & $\begin{array}{l}461,4 \\
783,9 \\
59,2 \\
94,8 \\
\end{array}$ & $\begin{array}{c}419,5 \\
871 \\
98,6 \\
105,4 \\
\end{array}$ & $\begin{array}{l}\text { SIM } \\
\text { SIM } \\
\text { NÂO } \\
\text { SIM }\end{array}$ & BOM \\
\hline Manaus-2 & $\begin{array}{l}\text { Proc. Básicos } \\
\text { Periodontia } \\
\text { Endodontia } \\
\text { Cirurgia }\end{array}$ & $\begin{array}{c}347,3 \\
566,8 \\
97,7 \\
212,6\end{array}$ & $\begin{array}{l}315,8 \\
629,7 \\
162,8 \\
236,2\end{array}$ & $\begin{array}{l}\text { SIM } \\
\text { SIM } \\
\text { SIM } \\
\text { SIM }\end{array}$ & ÓTIMO \\
\hline Manaus-3 & - & - & - & - & - \\
\hline Manaus-4 & $\begin{array}{l}\text { Proc. Básicos } \\
\text { Periodontia } \\
\text { Endodontia } \\
\text { Cirurgia }\end{array}$ & $\begin{array}{l}595,6 \\
652,5 \\
76,3 \\
69,4\end{array}$ & $\begin{array}{l}313,5 \\
435 \\
80,4 \\
40,8\end{array}$ & $\begin{array}{l}\text { SIM } \\
\text { SIM } \\
\text { NÂO } \\
\text { NÃO }\end{array}$ & REGULAR \\
\hline Maués & $\begin{array}{l}\text { Proc. Básicos } \\
\text { Periodontia } \\
\text { Endodontia } \\
\text { Cirurgia }\end{array}$ & $\begin{array}{l}330,5 \\
106,3 \\
35,8 \\
153,6\end{array}$ & $\begin{array}{c}413,1 \\
177,1 \\
102,1 \\
192 \\
\end{array}$ & $\begin{array}{l}\text { SIM } \\
\text { SIM } \\
\text { SIM } \\
\text { SIM }\end{array}$ & ÓTIMO \\
\hline Parintins & $\begin{array}{l}\text { Proc. Básicos } \\
\text { Periodontia } \\
\text { Endodontia } \\
\text { Cirurgia }\end{array}$ & $\begin{array}{l}216,3 \\
144 \\
64,4 \\
93,1 \\
\end{array}$ & $\begin{array}{c}196,7 \\
107,4 \\
160 \\
103,4\end{array}$ & $\begin{array}{l}\text { SIM } \\
\text { SIM } \\
\text { SIM } \\
\text { SIM }\end{array}$ & ÓTIMO \\
\hline $\begin{array}{l}\text { São Gabriel da } \\
\text { Cachoeira-1 }\end{array}$ & $\begin{array}{l}\text { Proc. Básicos } \\
\text { Periodontia } \\
\text { Endodontia } \\
\text { Cirurgia }\end{array}$ & $\begin{array}{l}58,8 \\
47,3 \\
76,3 \\
44,1\end{array}$ & $\begin{array}{l}73,4 \\
164 \\
135 \\
55,1\end{array}$ & $\begin{array}{l}\text { NÃO } \\
\text { SIM } \\
\text { SIM } \\
\text { NÂO }\end{array}$ & REGULAR \\
\hline $\begin{array}{l}\text { São Gabriel da } \\
\text { Cachoeira-2 }\end{array}$ & $\begin{array}{l}\text { Proc. Básicos } \\
\text { Periodontia } \\
\text { Endodontia } \\
\text { Cirurgia }\end{array}$ & $\begin{array}{c}124,6 \\
48,4 \\
73,2 \\
13,1\end{array}$ & $\begin{array}{c}113,3 \\
53,8 \\
121,9 \\
14,5\end{array}$ & $\begin{array}{c}\text { SIM } \\
\text { NÂO } \\
\text { SIM } \\
\text { NÂO }\end{array}$ & REGULAR \\
\hline Tefé & $\begin{array}{l}\text { Proc. Básicos } \\
\text { Periodontia } \\
\text { Endodontia } \\
\text { Cirurgia }\end{array}$ & $\begin{array}{c}32,2 \\
148,98 \\
2,8 \\
0\end{array}$ & $\begin{array}{c}40,2 \\
248,2 \\
236,7 \\
0\end{array}$ & $\begin{array}{l}\text { NÃO } \\
\text { SIM } \\
\text { SIM } \\
\text { NÃO }\end{array}$ & REGULAR \\
\hline
\end{tabular}

Fonte: SIA-SUS, 2006.

falhas na hierarquização dos serviços nos municípios do Amazonas. A falta da devida referência dos usuários à atenção secundária induz a menos opções de tratamento, maior índice de exodontias e consequente edentulismo na população, conforme evidenciado no último levantamento epidemiológico em saúde bucal (BRASIL, 2004a). Além disso, é importante ressaltar as características peculiares do Amazonas que dificultam a expansão da rede assistencial de saúde nas cidades do interior do estado. $\mathrm{O}$ isolamento geográfico e a falta de recursos financeiros que se concentram especialmente na capital, Manaus, seriam os mais importantes, os quais, aliados à má gestão em muitos dos municípios, contribuem para o baixo desempenho e qualidade dos serviços de saúde. Outra possibilidade seria a incoerência da meta estipulada pela portaria para o subgrupo de cirurgia, uma vez que estudo anterior conduzido em outra unidade federativa, no qual o presente estudo 
Tabela 4. Variáveis sociodemográficas dos municípios, cobertura da ESF e ESB e recursos humanos dos CEO, segundo o desempenho obtido no cumprimento das metas, Amazonas, Brasil, 2009

\begin{tabular}{|c|c|c|c|c|c|c|c|c|c|}
\hline $\begin{array}{l}\text { DESEM- } \\
\text { PENHO }\end{array}$ & & $\mathrm{IDH}^{*}$ & $\begin{array}{c}\text { PIB } \\
\text { per capita* }\end{array}$ & $\begin{array}{l}\text { Analfa- } \\
\text { betismo }\end{array}$ & $\begin{array}{c}\text { Incidência } \\
\text { de pobreza } \\
(\%)\end{array}$ & $\begin{array}{c}\text { Cobertura } \\
\left.\text { ESF* }^{*} \%\right)\end{array}$ & $\begin{array}{l}\text { Cobertura } \\
\left.\text { ESB* }^{*} \%\right)\end{array}$ & $\mathbf{N}^{\circ} \mathrm{CD} *$ & CD/ ASB* \\
\hline $\begin{array}{l}\text { ÓTIMO } \\
n=3\end{array}$ & $\begin{array}{l}\text { média } \\
\text { mínimo } \\
\text { máximo }\end{array}$ & $\begin{array}{l}0,717 \\
0,689 \\
0,774\end{array}$ & $\begin{array}{c}9501 \\
3518 \\
20894 \\
\end{array}$ & $\begin{array}{c}10,3 \\
6,1 \\
15,4 \\
\end{array}$ & $\begin{array}{l}52,10 \\
40,98 \\
60,07 \\
\end{array}$ & $\begin{array}{l}62,12 \\
32,74 \\
77,20 \\
\end{array}$ & $\begin{array}{l}37,01 \\
10,12 \\
65,52 \\
\end{array}$ & $\begin{array}{c}9 \\
4 \\
16 \\
\end{array}$ & $\begin{array}{c}1,51 \\
1 \\
1,77 \\
\end{array}$ \\
\hline $\begin{array}{l}\text { BOM } \\
n=1\end{array}$ & - & 0,774 & 20894 & 6,1 & 40,98 & 32,74 & 10,12 & 10 & 1,11 \\
\hline $\begin{array}{l}\text { REGULAR } \\
n=4\end{array}$ & $\begin{array}{l}\text { média } \\
\text { mínimo } \\
\text { máximo }\end{array}$ & $\begin{array}{l}0,696 \\
0,663 \\
0,774\end{array}$ & $\begin{array}{c}8304 \\
3621 \\
20894\end{array}$ & $\begin{array}{c}18,3 \\
6,1 \\
23,2\end{array}$ & $\begin{array}{l}47,86 \\
40,98 \\
61,64\end{array}$ & $\begin{array}{l}42,00 \\
32,74 \\
69,35\end{array}$ & $\begin{array}{l}26,80 \\
10,12 \\
37,34\end{array}$ & $\begin{array}{c}7,75 \\
3 \\
15\end{array}$ & $\begin{array}{c}1,71 \\
1 \\
3\end{array}$ \\
\hline $\begin{array}{l}\text { RUIM } \\
n=2\end{array}$ & $\begin{array}{l}\text { média } \\
\text { mínimo } \\
\text { máximo }\end{array}$ & $\begin{array}{l}0,646 \\
0,598 \\
0,694\end{array}$ & $\begin{array}{l}4708 \\
4639 \\
4776\end{array}$ & $\begin{array}{l}31,1 \\
20,8 \\
41,3 \\
\end{array}$ & $\begin{array}{l}62,28 \\
61,04 \\
63,52 \\
\end{array}$ & $\begin{array}{c}126,67 \\
70,06 \\
183,27 \\
\end{array}$ & $\begin{array}{c}102,64 \\
52,55 \\
152,73 \\
\end{array}$ & $\begin{array}{c}6,5 \\
5 \\
8 \\
\end{array}$ & $\begin{array}{c}1,33 \\
- \\
- \\
\end{array}$ \\
\hline $\begin{array}{l}\text { SEM } \\
\text { DADOS } \\
n=3\end{array}$ & $\begin{array}{l}\text { média } \\
\text { mínimo } \\
\text { máximo }\end{array}$ & $\begin{array}{l}0,699 \\
0,611 \\
0,774\end{array}$ & $\begin{array}{l}11225 \\
5569 \\
20894\end{array}$ & $\begin{array}{c}18,5 \\
6,1 \\
37,0 \\
\end{array}$ & $\begin{array}{l}46,04 \\
40,36 \\
56,78 \\
\end{array}$ & $\begin{array}{l}65,16 \\
32,74 \\
96,43 \\
\end{array}$ & $\begin{array}{l}52,47 \\
10,12 \\
81,00 \\
\end{array}$ & $\begin{array}{l}6 \\
4 \\
8 \\
\end{array}$ & $\begin{array}{c}1,73 \\
0,8 \\
2,66 \\
\end{array}$ \\
\hline
\end{tabular}

*Índice de Desenvolvimento Humano (IDH); Produto Interno Bruto (PIB); Estratégia de Saúde da Família (ESF); Equipes de Saúde Bucal (ESB); Cirurgiãodentista (CD); Auxiliar de saúde bucal (ASB).

Fonte: SIA-SUS, 2006

se fundamentou, também encontrou baixo percentual de cumprimento desta meta (FIGUEIREDO; GOES, 2009), considerando que apenas os procedimentos de maior complexidade devem ser referenciados aos CEO.

Da mesma forma, o desempenho não foi satisfatório para os procedimentos de endodontia, uma vez que $40 \%$ dos estabelecimentos não conseguiram cumprir a meta e $10 \%$ apresentaram valores limítrofes. Como tais procedimentos raramente são oferecidos na atenção básica, supõe-se mais uma vez que os problemas bucais referentes a esta especialidade estejam sendo solucionados neste nível de atenção por meio de exodontias. $\mathrm{O}$ entendimento de que a remoção dos dentes pode ser a solução para os problemas de saúde bucal pode estar também relacionado às características socioeconômicas e aos conceitos culturais ainda vigentes em nossa população (NATIONS; NUTO, 2002; SILVA; MAGALHÃES; FERREIRA, 2010).

Outras variáveis envolvidas na qualidade de serviços de saúde referem-se à estrutura física, humana e organizacional dos Centros de Especialidades Odontológicas. A regulamentação em vigor determina o número mínimo de um cirurgiâo-dentista e um auxiliar de saúde bucal por cadeira odontológica, sendo que o CEO deverá funcionar nos turnos matutino e vespertino e, durante esses períodos, sempre deverá haver profissionais de saúde à disposição no estabelecimento (BRASIL, 2006a). Quando analisada tal proporção (1:1) foi possível detectar que a mesma se faz presente em menos de $30 \%$ dos CEO avaliados, o que poderia diminuir a produtividade e a qualidade do serviço e aumentar o desgaste dos cirurgióes-dentistas (ORENHA; ELEUTÉRIO; SALIBA, 1998; KOVALESKI; BOING; FREITAS, 2005). Não ficou evidente nesse estudo uma relação entre tal desproporção e o baixo desempenho dos estabelecimentos, indicando que a produtividade dos serviços públicos pode estar sendo limitada por outros fatores.

Um estudo realizado em 2004 analisou o perfil de implantação da odontologia no Programa Saúde da Família no estado do Paraná (BALDANI et al., 2005). Dentre os resultados obtidos pelo estudo, os autores observaram que, quanto ao encaminhamento dos eventos de maior complexidade para os centros de referências, 9,5\% dos municípios do estado náo referenciam 
os casos, todavia os mesmos são solucionados pelas próprias equipes da saúde bucal. Foi constatado também que outros $9,5 \%$ dos municípios não resolvem os casos mais complexos e nem os referenciam, concluindo o estudo que existem problemas na referência para especialidades odontológicas, e destacando que os casos mais complexos também se tornam os de mais difícil solução. Os resultados coincidem com o presente estudo, pois a cobertura das equipes de saúde bucal e de saúde da família foi maior nos municípios com pior desempenho. De maneira contraditória com a metodologia operacional de reorganização do Sistema Único de Saúde, evidenciou-se que quanto maior a cobertura das equipes de saúde bucal na ESF, pior o desempenho do serviço. Este resultado é comparável ao de estudo anterior (FIGUEIREDO; GOES, 2009), que também não encontrou influência da alta cobertura na atenção básica com o bom desempenho da assistência especializada em saúde bucal.

As dificuldades de estruturação da saúde bucal à luz da ESF podem se dever ao fato de o perfil profissional estar voltado para a clínica, não para a promoção de saúde. Corroborando, há que se levar em conta a expectativa da comunidade, habituada ao atendimento curativo do profissional. Tais fatores refletem um atendimento meramente clínico e pontual, que contribuem para dificultar ou retardar a almejada mudança operacional no acesso à assistência odontológica e na forma da atenção à saúde bucal, conforme revelou estudo realizado no estado do Pernambuco (MARTELLI et al., 2008). Outro estudo mostrou que o percentual de evolução dos procedimentos odontológicos básicos realizados no período estudado foi incompatível ao percentual de expansão do número de ESB, o que, segundo os autores, leva a acreditar que, na prática, os serviços existentes de assistência odontológica para atenção básica foram transformados em equipes de saúde bucal (SILVA et al., 2011). Uma análise recente da implantação e impacto da ESF no estado do Amazonas indicou ausência de mudança no modelo assistencial, indicando não ser exclusividade das políticas de saúde bucal na atenção básica, mas que se apresenta na ESF como um todo (OLIVEIRA; GONÇALVES; PIRES, 2011).
Deve-se considerar, ainda, que a baixa capacidade de oferta dos serviços especializados no estado do Amazonas evidenciada pelo estudo dificulta o estabelecimento de sistemas adequados de referência e contrarreferência em saúde bucal na quase totalidade dos sistemas locorregionais de saúde, conforme estabelecido nas Diretrizes da Política Nacional de Saúde Bucal (BRASIL, 2004b).

Dentre os indicadores municipais avaliados, observou-se que quanto maior o IDH municipal, melhor foi o desempenho dos serviços, resultado concordante com o encontrado em estudo anterior (FIGUEIREDO; GOES, 2009). Como a taxa de alfabetizaçáo acima dos 15 anos e o rendimento per capita expandido são componentes que participam da construçáo do IDH, que envolve as dimensóes de educação, longevidade e renda, é razoável que as variáveis analfabetismo e PIB per capita também se distribuam acompanhando a relação do IDH com o cumprimento das metas, como se observa na síntese dos resultados segundo grupo de desempenho dos CEO.

O impacto dos fatores socioeconômicos e demográficos no contexto da avaliação dos serviços de saúde bucal tem sido amplamente descrito na literatura (FERNANDES; PERES, 2005; MATOS; GIATTI; LIMACOSTA, 2004; BARBATO et al., 2007; ANTUNES, 2008). Também indicou-se que a extração dentária é característica da atenção odontológica em saúde públi$\mathrm{ca}$, em especial de municípios que apresentam piores indicadores socioeconômicos (FERNANDES; PERES, 2005; BARBATO et al., 2007).

Apesar da inegável expansão, a lenta reorganização do modelo de atenção odontológica em saúde pública fica evidente quando analisamos as recomendaçóes da II Conferência Nacional de Saúde Bucal, realizada em 1993, que já sugeriam a implementação de políticas econômicas e sociais que possam repercutir favoravelmente sobre as condições de saúde da população, a desarticulação entre os três níveis de governo e definição de um modelo assistencial que contemple com clareza a prestação de serviços odontológicos, o acesso à educação e a transferência do conhecimento para a populaçáo como uma preocupação essencial no processo de resgate da cidadania dos brasileiros, alteração da 
forma inadequada de repasse dos recursos financeiros da União para os municípios, que, além de permitirem a sua apropriação pelos estados, baseiam-se exclusivamente na produtividade dos serviços e não no planejamento que contemple o perfil epidemiológico e a qualidade dos serviços prestados (CONSELHO FEDERAL DE ODONTOLOGIA, 1993). Os resultados encontrados nesse estudo revelam que as mesmas questóes perpetuaram-se e um grande amadurecimento do sistema fazia-se necessário para uma maior cobertura e efetividade da assistência especializada em saúde bucal.

Por se tratar de um número reduzido de estabelecimentos, somente foi possível uma análise descritiva, mas que se revelou capaz de apresentar a situação dos CEO no Amazonas. A inclusão dos estabelecimentos com pouco tempo de habilitação, ou daqueles que não a possuíam, também pode ter influenciado os resultados do estudo, uma vez que as dificuldades operacionais na fase inicial de implantação do serviço podem interferir na sua produção ambulatorial. Além disso, devem ser consideradas as limitações inerentes à natureza da informação, uma vez que os dados secundários estão sujeitos a sub-registros, erros de classificação ou mesmo ao despreparo administrativo e às dificuldades de transmissão dos dados em municípios com menos recursos.

\section{Conclusões}

A atenção secundária em saúde bucal no estado do Amazonas não apresentou um desempenho satisfatório, no período estudado, quanto ao cumprimento das metas de produçáo ambulatorial, sendo que um estabelecimento do município de Manaus e os estabelecimentos de Maués e Parintins apresentaram os melhores desempenhos. Parece, ainda, não haver relação entre o desempenho avaliado e a cobertura das equipes de saúde bucal da Estratégia de Saúde da Família, número de cirurgiôes-dentistas e proporção de auxiliar de saúde bucal/cirurgióes-dentistas no centro de especialidades, destarte, os resultados sugerem que os desempenhos dos CEO podem estar mais ligados às características sociodemográficas dos municípios do que com as características estruturais dos serviços.

\section{Referências}

ANTUNES, J. L. F. Condições socioeconômicas em saúde: discussão de dois paradigmas. Revista de Saúde Pública, São Paulo, v. 42, n. 3, p. $562-567,2008$

BALDANI, M. H. et al. A inclusão da odontologia no Programa Saúde da Família no estado do Paraná, Brasil. Cadernos de Saúde Pública, Rio de Janeiro, v. 21, n. 4, p. 1026-1035, 2005.

BARBATO, P. R. et al. Perdas dentárias e fatores sociais, demográficos e de serviços associados em adultos brasileiros: uma análise dos dados do Estudo Epidemiológico Nacional (Projeto SB Brasil 20022003). Cadernos de Saúde Pública, Rio de Janeiro, v. 23, n. 8, p. 18031814, 2007.

BRASIL. Ministério da Saúde. Portaria no 1.329, de 12 de novembro de 1999. Estabelece nova sistemática para o cálculo do incentivo financeiro ao Programa de Saúde da Família, parte integrante do Piso da Atenção Básica - PAB. Brasília: Diário Oficial da União, 16 nov. 1999. p. 23. Seção 1.
Portaria no 673, de 03 de junho de 2003. Atualiza e revê o incentivo financeiro às Ações de Saúde Bucal, no âmbito do Programa de Saúde da Família, parte integrante do Piso de Atenção Básica - PAB. Brasília: Diário Oficial da União, 04 jun. 2003. p. 44. Seção 1.

Portaria no 1750, de 29 de julho de 2004. Estabelece critérios, normas e requisitos para a implantação e habilitação de Centros de Especialidades Odontológicas e Laboratórios Regionais de Próteses Dentárias. Brasília: Diário Oficial da União, 30 jul. 2004c. p. 71. Seção 1.

Portaria no 599, de 23 de março de 2006. Define a implantação de Centros de Especialidades Odontológicas (CEO) e de Laboratórios Regionais de Próteses Dentárias (LRPDs) e estabelece critérios, normas e requisitos para seu credenciamento. Brasília: Diário Oficial da União, 24 mar. 2006a. p. 51. Seção 1 . 
Portaria no 600, de 23 de março de 2006. Institui o financiamento dos Centros de Especialidades Odontológicas. Brasília: Diário Oficial da União, 24 mar. 2006b. p. 52. Seção 1.

Ministério da Saúde; ORGANIZAÇÃO PAN-AMERICANA DA SAÚDE. Painel de indicadores do SUS no 7: panorâmica VIII. Brasília: OPAS, 2010.

Ministério da Saúde. Secretaria de Atenção à Saúde. Departamento de Atenção Básica. Coordenação Nacional de Saúde Bucal. Projeto SB Brasil 2003: condições de saúde bucal da população brasileira 2002-2003: Resultados principais. Brasília: Ministério da Saúde, 2004a.

Diretrizes da Política Nacional de Saúde Bucal. Brasília: Ministério da Saúde, 2004b. Disponível em: <http://portal.saude. gov.br/portal/arquivos/pdf/politica_nacional_brasil_sorridente. pdf > . Acesso em: 23 out. 2010.

CONSELHO FEDERAL DE ODONTOLOGIA (Brasil). Relatório Final. In: Conferência Nacional de Saúde Bucal, 2., 1993, Brasília. Brasília: Conselho Federal de Odontologia, 1993.

FERNANDES, L. S.; PERES, M. A. Associação entre atenção básica em saúde bucal e indicadores socioeconômicos municipais. Revista de Saúde Pública, São Paulo, v. 39, n. 6, p. 930-936, 2005.

FIGUEIREDO, N.; GOES, P. S. A. Construção da atenção secundária em saúde bucal: um estudo sobre os Centros de Especialidades Odontológicas em Pernambuco, Brasil. Cadernos de Saúde Pública, Rio de Janeiro, v. 25, n. 2, p. 259-267, 2009.

KOVALESKI, D. F.; BOING, A. F.; FREITAS, S. F. T. Recursos humanos auxiliares em saúde bucal: retomando a temática. Revista de Odontologia da UNESP, São Paulo, v. 34, n. 4, p. 161-165, 2005.

MARTELLI, P. J. L. et al. Análise do modelo de atenção à saúde bucal em municípios do estado de Pernambuco. Ciência \& Saúde Coletiva, Rio de Janeiro, v. 13, n. 5, p. 1669-1674, 2008.
MATOS, D. M.; GIATTI, L.; LIMA-COSTA, M. F. Fatores sociodemográficos associados ao uso de serviços odontológicos entre idosos brasileiros: um estudo baseado na Pesquisa Nacional por Amostra de Domicílios. Cadernos de Saúde Pública, Rio de Janeiro, v. 20, n. 5, p. 1290-1297, 2004.

NATIONS, N. K.; NUTO, S. A. S. Tooth worms, poverty tatoos and dental care conflicts in Northeast Brazil. Social Science \& Medicine, New York, v. 54, n. 2, p. 229-234, 2002.

OLIVEIRA, H. M.; GONÇALVES, M. J. F.; PIRES, R. O. M. Caracterização da estratégia saúde da família no estado do Amazonas, Brasil: análise da implantação e impacto. Cadernos de Saúde Pública, Rio de Janeiro, v. 27, n. 1, p. 35-45, 2011.

ORENHA, E. S.; ELEUTÉRIO, D.; SALIBA, N. A. Organização do atendimento odontológico no serviço público: trabalho auxiliado, produtividade e ambiente físico. Revista de Odontologia da UNESP, São Paulo, v. 27, n. 1, p. 215-224, 1998.

ORGANIZAÇÃO PAN-AMERICANA DA SAÚDE; BRASIL. Ministério da Saúde. A Política Nacional de Saúde Bucal do Brasil: registro de uma conquista histórica. Série Técnica Desenvolvimento de Sistemas e Serviços de Saúde, 11. Brasília: Organização PanAmericana da Saúde, 2006. Disponível em: <http://dab.saude. gov.br/docs/publicacoes/geral/serie_tecnica_11_port.pdf $>$. Acesso em: 12 jan. 2011.

PINHEIRO, R. S.; TORRES, T. Z. G. Uso de serviços odontológicos entre os estados do Brasil. Ciência \& Saúde Coletiva, Rio de Janeiro, v. 11, n. 4, p. 999-1010, 2006.

SILVA, M. E. S.; MAGALHÃES, C. S.; FERREIRA, E. F. Perda dentária e expectativa da reposição protética: estudo qualitativo. Ciência \& Saúde Coletiva, Rio de Janeiro, v. 15, n. 3, p. 813-820, 2010.

SILVA, S. F. et al. Análise do avanço das equipes de saúde bucal inseridas na estratégia saúde da família em Pernambuco, Região Nordeste, Brasil, 2002 a 2005. Ciência \& Saúde Coletiva, Rio de Janeiro, v. 16, n. 1, p. 211-220, 2011.

Recebido para publicação em Outubro/2011

Versão final em Julho/2012

Conflito de interesse: Não houve

Suporte financeiro: Inexistente 